\title{
Caractéristiques Physicochimiques Récoltés des Miels de la Sous-Préfecture de Cechi (Dans le Département D’Agboville, Côte D’Ivoire)
}

\section{Kouamé Koffi Félix}

Doctorant au Laboratoire des Milieux Naturels et Conservation de la Biodiversité, UFR Biosciences, Université Félix Houphouët-Boigny,

Côte d'Ivoire

\section{Gbouhoury Eric-Kevin Bolou}

Chargé de recherche au Centre National de Floristique, UFR Biosciences, Université Félix Houphouët-Boigny, Côte d'Ivoire

Fofié N'Guessan Bra Yvette

Enseignant-chercheur au Laboratoire de Pharmacognosie Botanique et Cryptogamie, UFR Sciences Pharmaceutiques et Biologiques, Université Félix Houphouët-Boigny, Côte d'Ivoire

\section{Kassi N'Dja Justin}

Professeur Titulaire au Laboratoire des Milieux Naturels et Conservation de la Biodiversité, UFR Biosciences, Université Félix Houphouët-Boigny,

Côte d'Ivoire

\section{Doi:10.19044/esj.2021.v17n34p286}

Submitted: 11 May 2021

Accepted: 02 September 2021

Published: 30 September 2021
Copyright 2021 Author(s)

Under Creative Commons BY-NC-ND 4.0 OPEN ACCESS

Cite As:

Kouamé K.F., Gbouhoury E-K. B., Fofié N’Guessan B.Y. \& Kassi N’Dja J. (2021). Caractéristiques Physicochimiques Récoltés des Miels de la Sous-Préfecture de Cechi (dans le Département D’Agboville, Côte D’Ivoire). European Scientific Journal, ESJ, 17(34), 286. https://doi.org/10.19044/esj.2021.v17n34p286

\section{Résumé}

La caractérisation des miels en développement d'un pays est nécessaire pour leur valorisation. Cette étude est une contribution à la connaissance de la qualité des miels des forêts de la Côte d'Ivoire. Des échantillons de miel de cinq localités de la Sous-Préfecture de Cechi (Agboville) ont été étudiés. Les paramètres physicochimiques des miels ont été analysés pour vérifier leur conformité aux normes du Codex alimentarius. Ces analyses ont donné les valeurs moyennes suivantes : le pH est 3,55 $\pm 0,46$; la conductivité électrique $(\sigma)$ est $98,01 \pm 31 \mu \mathrm{S} / \mathrm{cm}$, l'acidité libre est 
$19,50 \pm 4,47 \mathrm{mEq} / \mathrm{Kg}$; la densité relative est $1,42 \pm 0,07$; la teneur en eau est de $20,2 \pm 3,11 \%$, les cendres représentent $0,18 \pm 0,11 \%$ et la teneur en sucre totale est de 78,31 $\pm 0,19 \%$. La plupart de ces paramètres sont conformes au Codex alimentarius, à l'exception des teneurs en sucres qui dépasse la limite maximale accepté. Cela traduit que les miels analysés sont issus de nectar trop sucrés.

Mots clés : Miel, Analyse Physicochimique, Codex Alimentarius, Zone Forestière, Côte d'Ivoire

\title{
Physicochemical Characteristics of Honey Collected from The Sub-Prefecture of Cechi (In The Department of Agboville, Ivory Coast)
}

\section{Kouamé Koffi Félix}

PhD Student, Laboratory of Natural Environments and Biodiversity Conservation, UFR Biosciences, Felix Houphouët-Boigny University, Ivory Coast

\section{Gbouhoury Eric-Kevin Bolou}

Researcher, National Floristic Center, UFR Biosciences, Felix HouphouëtBoigny University, Ivory Coast

Fofié N'Guessan Bra Yvette

Lecturer-researcher at the Laboratory of Botanical Pharmacognosy and Cryptogamy, UFR Pharmaceutical and Biological Sciences, Felix Houphouët-Boigny University, Ivory Coast

\section{Kassi N'Dja Justin}

Full Professor, Laboratory of Natural Environments and Biodiversity Conservation, UFR Biosciences, Felix Houphouët-Boigny University, Ivory Coast

\begin{abstract}
The characterisation of a country's developing honeys is necessary for their valorisation. This study is a contribution of the knowledge of the Ivory Coast forests honeys quality. Honey samples from five localities of the SubPrefecture of Cechi (Agboville) were studied. The physicochemical parameters of the honeys were analyzed to verify their conformity to the standards of the Codex alimentarius. These analyses yielded the following average values: $\mathrm{pH}$ is $3.55 \pm 0.46$; electrical conductivity $(\sigma)$ is $98.01 \pm 31$ $\mu \mathrm{S} / \mathrm{cm}$, free acidity is $19.50 \pm 4.47 \mathrm{mEq} / \mathrm{Kg}$; relative density is $1.42 \pm 0.07$; water content is $20.2 \pm 3.11 \%$, ash represents $0.18 \pm 0.11 \%$ and total sugar content is $78.31 \pm 0.19 \%$. Most of these parameters are in conformity with the
\end{abstract}


Codex Alimentarius, except for the sugar content which exceeds the maximum accepted limit. This means that the analyzed honeys are made from nectar with too much sugar.

Keywords: Honey, Physicochemical Analysis, Codex Alimentarius, Forest Area, Ivory Coast

\section{Introduction}

Le miel est une substance sucrée d'origine naturelle produite par l'espèce Apis mellifera à partir du nectar de plantes ou à partir de secrétions provenant des parties vivantes de plantes ou à partir d'excrétions d'insectes butineuses laissées sur les parties vivantes des plantes, que les abeilles butinent, transforment en les combinent avec des substances spécifiques qu'elles sécrètent elle-même, déposent, déshydratent, emmagasinent et laissent affiner et mûrir dans les rayons de la ruche (Codex, 2001; Randrianarivelo, 2010 ; Abdallah et Saber, 2017).

Le miel est consommé par l'homme et les animaux sauvages en tant qu'aliment (Kombo, 1989). Il est aussi utilisé dans la composition de plusieurs produits pharmaceutiques et comme médicament pour le traitement de certaines maladies et affections (Mehnaz et Bonmatin, 2003 ; Ahouandjinou et al., 2016 ; Assi-Kaudjhis et al., 2020). Par exemple, il est antianémique, antiseptique, diurétique, énergétique, fébrifuge et antitussif ; il soulage les angines, la bronchite et facilite la cicatrisation des blessures (Maglon et Vanwijek, 2003 ; Bradbear, 2005).

Depuis l'antiquité le miel est recherché par l'homme ; ses multiples propriétés thérapeutiques et nutritionnelles ont fait évoluer la production du miel de la cueillette à l'élevage des abeilles. Le miel est composé d'hydrates de carbone, des acides organiques, des acides aminés, des protéines, des minéraux, des vitamines et des lipides qui varient selon les plantes butinées, le climat, les conditions environnementales (Makhtar, 2002 ; Mezhoud, 2013).

Le caractère du miel dépend des plantes visitées par les abeilles. Selon N'guessan et al. (2009), les effets thérapeutiques du miel sont dus aux flavonoïdes contenus dans les drogues végétales. Ainsi, le miel doit sa coloration aux pigments tels que les caroténoïdes et les flavonoïdes, qui varient selon son origine géographique et florale (Randrianarivelo, 2010). L'analyse du miel consiste à la mesure de ses paramètres physicochimiques et à la recherche de ses origines botaniques et géographiques (Makhloufi et al., 2013). En Côte d'Ivoire, les études sur l'analyse physicochimique des miels concernent des localités du Centre (Iritié et al., 2014 ; Assi Kaudjhis et al., 2021) et du Nord (Coulibaly, 2014 ; Diomandé et al., 2018). Ces régions sont caractérisées par des savanes préforestières et sèches. Or sur un total de 3853 espèces végétales vasculaires répertoriées en Côte d'Ivoire, $3 / 4 \quad$ sont 
essentiellement forestières (Aké-Assi, 2002). Cela représente un grand potentiel mellifère dans le Sud forestier ivoirien. Ce travail sur l'analyse physicochimique des miels de la Sous-Préfecture de Cechi dans la zone forestière se situe dans le cadre de la promotion des miels de forêt.

\section{Matériel et méthodes \\ Milieu d'étude}

Les différentes localités d'échantillonnages et la réserves sont situées dans la Sous-Préfecture de Cechi, dans le Département d'Agboville au SudEst de la Côte d'Ivoire (Figure 1). Le Département d'Agboville, appartient à la région administrative de l'Agneby-Tiassa. C'est une région du domaine guinéen de la Côte d'Ivoire (Aubréville, 1958). Elle est caractérisée par quatre saisons (deux saisons pluvieuses et deux saisons sèches) avec une pluviométrie moyenne annuelle de 1585,35 mm et une température moyenne annuelle de $26,72^{\circ} \mathrm{C}$ (Kouamé et al. 2020). La population de la SousPréfecture de Cechi est estimée à 22.779 habitants (INS, 2014). Les Abbey et les Krobou sont les peuples autochtones. Les migrants sont originaires des autres régions de la Côte d'Ivoire (Agny, Baoulé, Koulango, Malinké) et des pays frontaliers (Burkinabé, Malien, Guinéen). L’agriculture et le commerce sont les principales activités de cette population (Kouamé et al., 2020).

\section{Echantillonnage}

Au total 5 échantillons de miel de la Sous-Préfecture de Cechi de l'année 2020 ont été utilisés pour cette étude. Quatre des échantillons de miel sont issus de la cueillette des ruches naturelles, collectés auprès des agriculteurs des localités d'Allany (E1) ; Banguié 2 (E2) ; Mitichi (E4) ; N'guessan Blekro (E5) et l'échantillon de miel des ruches langstroths installées dans la réserve (E3) à BouaM'po (Figure 2). La figure 1 présente les localités de collecte et la réserve naturelle dans la Sous-Préfecture de Cechi dans le Département d’Agboville. 


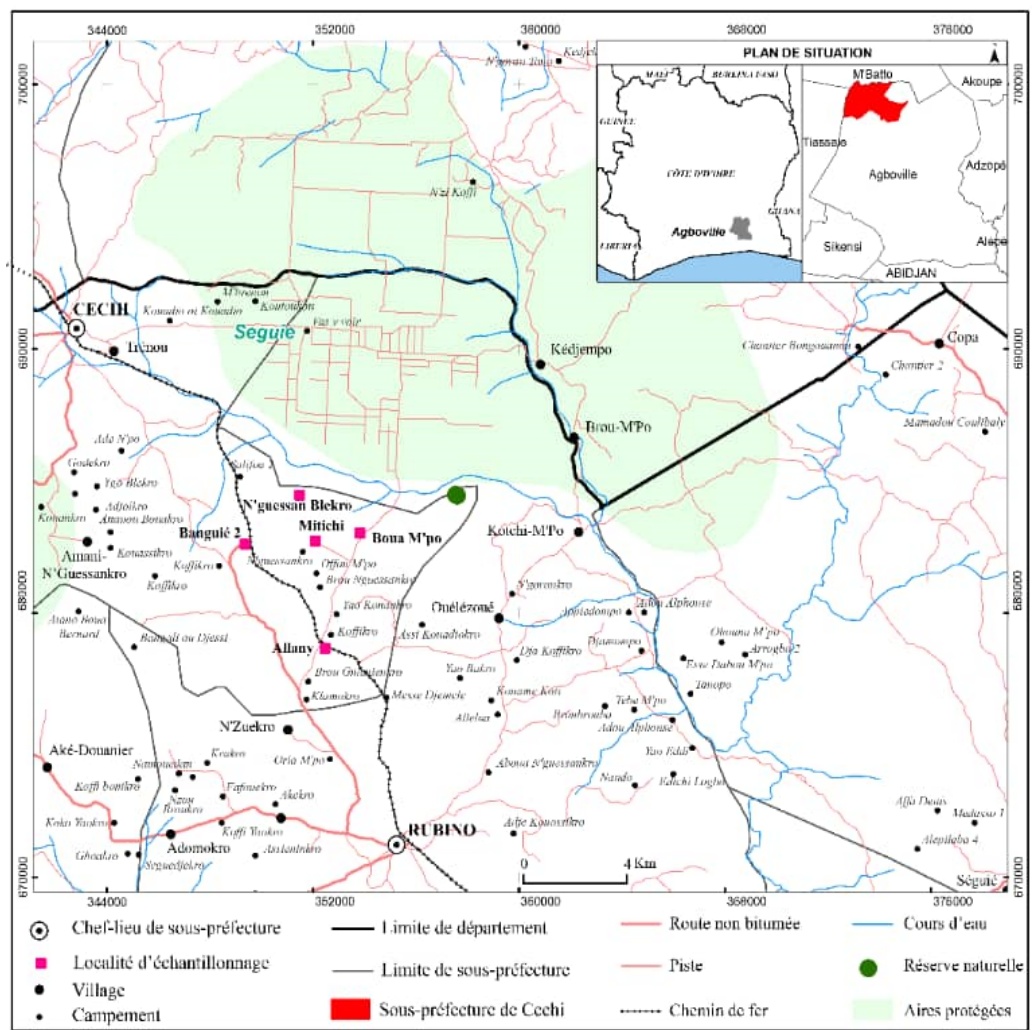

Figure 1. Situation géographique des localités d’échantillonnage (Sous-Préfecture de Cechi)

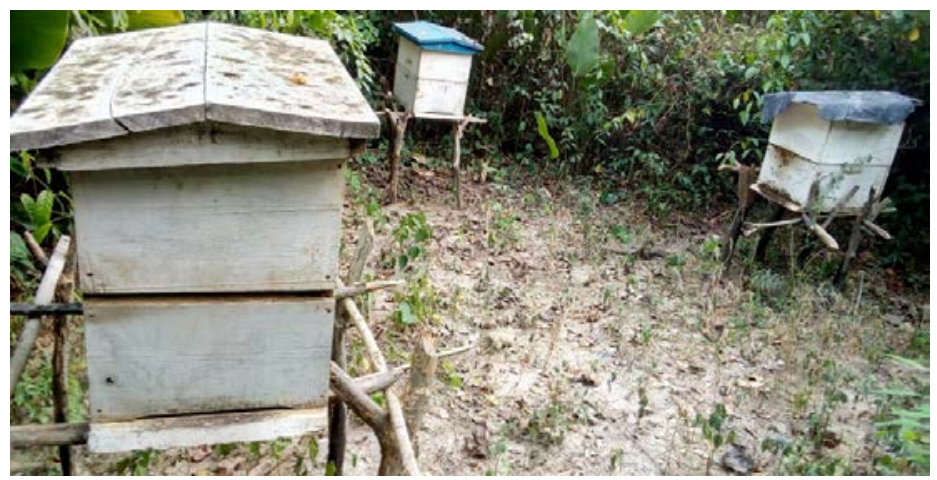

Figure 2. Aperçus des ruches langstroths dans la réserve naturelle (Kouamé et al., 2021)

\section{Analyse physicochimique des miels de la Sous-Préfecture de Cechi}

Les analyses ont été réalisées au sein du service de contrôle des aliments du Laboratoire Nationale de Santé Publique (LNSP) de Treichville en Côte d'Ivoire. Les paramètres ont été déterminés selon les protocoles suivants : Le pH, par dilution de $10 \mathrm{mg}$ de miel à l'eau distillée dans un bécher 
de $100 \mathrm{ml}$ et soumis à ultra-son pendant 15 minutes. La lecture s'est faite en plongeant les électrodes du $\mathrm{pH}$-mètre dans la solution.

La conductivité électrique est obtenue à $20^{\circ} \mathrm{C}$ en introduisant les électrodes du pH-mètre et du conductimètre dans la solution de miel diluée à $1 / 10$ ème. Les valeurs sont données en microsiemens par centimètre $(\mu \mathrm{S} / \mathrm{cm})$.

Le degré Brix et la teneur en eau sont déterminés en mettant une goutte de miel sur la plaque transparente du refractomètre, après étalonnage à l'eau distillée. Les lectures sont faites à l'écran à $20^{\circ} \mathrm{C}$.

La teneur en cendres par incinération d'une prise d'essai dans un four à $550^{\circ} \mathrm{C}$, puis la pesée du résidu obtenu (Assi-Kaudjhis et al., 2021). Les valeurs sont exprimées en pourcentage (\%) et donnée par la formule suivante :

$$
\text { Teneur cendre }(\%)=\frac{\mathrm{m} 2-\mathrm{m} 0}{\mathrm{~m} 1-\mathrm{m} 0} \times 100
$$

$\mathbf{m}_{\mathbf{0}}$ : masse en gramme des capsules vides $; \mathbf{m}_{\mathbf{1}}$ : masse en gramme des capsules de prises d'essai ; $\mathbf{m}_{2}$ : masse en gramme des capsules de prises d'essai après incinération.

L'acidité libre a été déterminée en neutralisant $25 \mathrm{ml}$ de miel dilué à $1 / 10$ ème avec du $\mathrm{NaOH}(0.05 \mathrm{~N})$ (Amri, 2006). Les valeurs sont exprimées en $\mathrm{mEq} / \mathrm{Kg}$ et données par la relation suivante :

$$
\text { Acidité libre }(\mathrm{mEq} / \mathrm{Kg})=\frac{1000 * \mathrm{~V} * 0,05}{\mathrm{PE}} \times 10
$$

V : Volume de soude $(\mathrm{NaOH})$ versé ; PE : Prise d'essai de miel utilisée.

La densité est obtenue en calculant le quotient de la masse volumique du miel et de celle de l'eau (Coulibaly, 2014 ; Assi-Kaudjhis et al., 2021 ; Koudegnan et al., 2021). Elle est donnée par la relation suivante :

$$
\mathbf{d}=\boldsymbol{\rho m} / \boldsymbol{\rho e}
$$

d : densité du miel ; pm: masse volumique du miel ; pe: masse volumique de l'eau pure

L’état de fermentation des miels et leur origine ont été déterminés.

\section{Analyse statistique}

Les moyennes des données des paramètres physicochimiques ont été soumises à des tests statistiques à l'aide du logiciel XLSTAT 2014.5.03, afin de vérifier si les cinq échantillons de miel sont statistiquement différents. Les variances des données sont comparées à l'aide du test statistique de Bartlett (Addinsoft, 2020).

\section{Résultats et discussion}

Valeurs des paramètres physico-chimiques des miels

Les valeurs obtenues des paramètres physicochimiques des miels sont consignées dans le tableau 1. 
Tableau 1.Caractéristiques physicochimiques des miels de la Sous-Préfecture de Cechi

\begin{tabular}{|c|c|c|c|c|c|c|c|}
\hline \multirow{2}{*}{$\begin{array}{c}\text { Echantillons } \\
\text { de miel }\end{array}$} & \multicolumn{7}{|c|}{ Paramètres physicochimiques \pm écart-types } \\
\hline & $\mathrm{pH}$ & $\begin{array}{c}\text { Acidité } \\
\text { libre } \\
(\mathrm{mEg} / \mathrm{Kg})\end{array}$ & Densité & $\begin{array}{c}\text { Teneur en } \\
\text { eau (\%) }\end{array}$ & Degré Brix (\%) & $\begin{array}{c}\text { Teneur en } \\
\text { cendre (\%) }\end{array}$ & $\begin{array}{c}\text { Conductivité } \\
\text { électrique }(\sigma) \\
(\mu \mathrm{S} / \mathrm{cm})\end{array}$ \\
\hline E1 & $3,40 \pm 0,02$ & $15,00 \pm 0,00$ & $1,40 \pm 0.01$ & $20 \pm 0,1$ & $76,67 \pm 0,06$ & $0,22 \pm 0,01$ & $124,6 \pm 0,00$ \\
\hline E2 & $4,34 \pm 0,56$ & $15,83 \pm 1,44$ & $1,39 \pm 0,02$ & $21 \pm 0,4$ & $76,50 \pm 0,10$ & $0,12 \pm 0,01$ & $53,80 \pm 0,17$ \\
\hline E3 & $3,26 \pm 0,02$ & $23,33 \pm 2,89$ & $1,47 \pm 0,03$ & $18 \pm 1,05$ & $78,90 \pm 0,00$ & $0,13 \pm 0,02$ & $96,00 \pm 0,00$ \\
\hline E4 & $3,22 \pm 0,03$ & $25,00 \pm 0,00$ & $1,33 \pm 0,05$ & $25 \pm 2,4$ & $78,10 \pm 0,06$ & $0,09 \pm 0,00$ & $86,30 \pm 0,10$ \\
\hline E5 & $3,52 \pm 0,06$ & $18,33 \pm 2,89$ & $1,5 \pm 0,04$ & $17 \pm 1,6$ & $81,40 \pm 0,00$ & $0,36 \pm 0,02$ & $129,47 \pm 0,38$ \\
\hline Moyennes & $3,55 \pm 0,46$ & $19,50 \pm 4,47$ & $1,42 \pm 0,07$ & $20,2 \pm 3,11$ & $78,31 \pm 0,19$ & $0,18 \pm 0,11$ & $98,01 \pm 31$ \\
\hline $\begin{array}{c}\text { Norme } \\
\text { Codex } \\
\text { alimentarius } \\
(2000 ; \\
\mathbf{2 0 0 1 )}\end{array}$ & $\begin{array}{l}3,5-4,5] \\
\text { Miel de } \\
\text { nectar; } \\
{[5-5,5]} \\
\text { Miel de } \\
\text { miellat }\end{array}$ & $\begin{array}{c}\leq 50 \\
\mathrm{mEq} / \mathrm{Kg}, \\
\text { tous les } \\
\text { miels }\end{array}$ & $\begin{array}{c}{[1,39-} \\
1,44] \text { tous } \\
\text { les miels }\end{array}$ & $\begin{array}{c}\leq 21 \% \text { tous } \\
\text { les miels } \\
\text { sauf } \\
\text { exception }\end{array}$ & $\begin{array}{c}\leq 65 \% \text { Miel de } \\
\text { nectar } ; \leq 45 \% \\
\text { Miel de miellat } \\
; \leq 53 \% \text { Miel de } \\
\text { mélange }\end{array}$ & $\begin{array}{c}\leq 0,6 \% \text { tous } \\
\text { les miels } \\
\text { sauf } \\
\text { exception }\end{array}$ & $\begin{array}{c}\leq 0,8 \mathrm{mS} / \mathrm{cm} \\
\text { Miel de nectar } \\
; \geq 1,2 \mathrm{mS} / \mathrm{cm} \\
\text { Miel de } \\
\text { miellat }\end{array}$ \\
\hline
\end{tabular}

NB: $1 \mu \mathrm{S} / \mathrm{cm}=10$ exp-4 mS/cm

\section{Potentiel d'Hydrogène ( $\mathrm{pH}$ ) des miels}

Les $\mathrm{pH}$ varient de 3,22 $\pm 0,03$ (miel de Mitichi, E4) à 4,34 $\pm 0,56$ (miel de Banguié 2) avec une moyenne de 3,55 $\pm 0,46$. Les échantillons de miels E1, $\mathrm{E} 3$ et $\mathrm{E} 4$ ont un $\mathrm{pH}$ inférieur à 3,5. Les échantillons de miel E2 et E5 ont un $\mathrm{pH}$ compris entre 3,5 et 4,5. Les $\mathrm{pH}$ des miels sont statistiquement identiques $(\mathrm{p}=0,406)$.

\section{Acidité libre des miels}

L'acidité libre varie de $15,00 \pm 0,00 \mathrm{mEq} / \mathrm{Kg}$ (miel d'Allany, E1) à $25,00 \pm 0,00 \mathrm{mEq} / \mathrm{Kg}$ (miel de Mitichi, E4) avec une moyenne de 19,50 \pm 4,47 $\mathrm{mEq} / \mathrm{Kg}$. Les acidités sont inférieures à $50 \mathrm{mEq} / \mathrm{Kg}$. Les acidités libres des miels sont statistiquement identiques $(p=0,406)$.

\section{Densité des miels}

La densité se situe entre 1,33 $\pm 0,05$ (miel de Mitichi E4) et 1,5 $\pm 0,04$ (miel de N'guessan Blekro, E5) avec une moyenne de 1,42 $\pm 0,07$. Les échantillons E3 et E5 ont une densité supérieure à 1,44. Les densités des miels sont statistiquement identiques $(\mathrm{p}=0,406)$.

\section{Teneur en eau ou humidité des miels}

La teneur en eau oscille de $17 \pm 1,6 \%$ à $25 \pm 2,4 \%$ avec une moyenne de 20,2 $\pm 3,11 \%$. La plupart des miels ont une teneur en eau inférieure ou égale à $21 \%$ sauf le miel de Mitichi (E4). Les teneurs en eau des miels sont statistiquement identiques $(\mathrm{p}=0,406)$. 


\section{Degré Brix ou teneur en sucre totale des miels}

Le degré Brix se situe entre 76,50 $\pm 0,10 \%$ (miel de Banguié 2, E2) à $81,40 \pm 0,00 \%$ (miel de N'guessan Blekro, E5) avec une moyenne de 78,31 \pm $0,19 \%$. Les miels ont une teneur en sucre totale supérieure à $65 \%$. Les degrés Brix des miels sont statistiquement identiques $(p=0,406)$.

\section{Teneur en cendre des miels}

La teneur en cendre varie de $0,09 \pm 0,00 \%$ (miel de Mitichi, E4) à 0,36 $\pm 0,02 \%$ (miel de N'guessan Blekro, E5) avec une moyenne de 0,18 $\pm 0,11 \%$. La teneur en cendre des miels est inférieure à $0,6 \%$. Les teneurs en cendre des miels sont statistiquement identiques $(\mathrm{p}=0,406)$.

\section{Conductivité électrique ( $\sigma$ ) des miels}

La conductivité électrique se situe entre 53,80 $\pm 0,17 \mu \mathrm{S} / \mathrm{cm}$ (miel de Banguié 2, E2) et $129,47 \pm 0,38 \mu \mathrm{S} / \mathrm{cm}$ (miel de N'guessan Blekro, E5) avec une moyenne de $98,03 \pm 31 \mu \mathrm{S} / \mathrm{cm}$. Ces valeurs sont inférieures à $0,8 \mathrm{mS} / \mathrm{cm}$. Les conductivités électriques des miels sont statistiquement identiques $(\mathrm{p}=$ $0,406)$.

\section{Interprétation des valeurs des paramètres physicochimiques}

Les analyses statistiques effectuées n’ont montré aucune différence significative entre les miels étudies ( $p>0,05)$.

\section{Potentiel d'Hydrogène (pH) des miels}

Les miels de la Sous-Préfecture de Cechi sont des miels acides car leurs $\mathrm{pH}$ se situent entre 3,22 $\pm 0,03$ et 4,34 $\pm 0,56$. Ces valeurs de $\mathrm{pH}$ de l'étude sont en conformité avec celles des miels malaisiens ( $\mathrm{pH}: 2,51$ et 3,26$)$ issus des travaux de Julika et al. (2019) et dans les miels nigérians ( $\mathrm{pH}: 3,10$ et 3,48) réalisés par Agbagwa et al. (2011). Selon le Codex alimentarius (2000 ; 2001), les miels de nectar ont un $\mathrm{pH}$ compris entre 3,5 et 4,5. Or tous les miels de la Sous-Préfecture de Cechi se trouvent dans cet intervalle. Donc les miels de la Sous-Préfecture de Cechi pourraient être des miels de nectar.

Les variations des $\mathrm{pH}$ observées d'une localité à une autre pourraient se justifier par les origines florales des miels, par le processus de formation du miel par les abeilles, par la méthode d'extraction du miel, et par la technique de conservation du miel (Louveaux, 1985 ; Achour et Khalil, 2014 ; Rebiai et al., 2015 et Selvaraju et al., 2019). Par ailleurs, les miels acides inhibent mieux la croissance et la prolifération des microorganismes; présentant ainsi un meilleur pouvoir antibactérien pour le traitement des plaies (Julika et al., 2019). 


\section{Acidité libre des miels}

L'acidité libre des miels varie de $15,00 \pm 0,00 \mathrm{mEq} / \mathrm{Kg}$ à $25,00 \pm 0,00$ $\mathrm{mEq} / \mathrm{Kg}$. Ces valeurs sont inférieures à la valeur limite $(50 \mathrm{mEq} / \mathrm{Kg}) \mathrm{du}$ Codex alimentarius (2000; 2001). Elles indiquent l'absence de fermentations indésirables dans les miels. Les miels pourraient être conservés longtemps dans des conditions appropriées sans risque de fermentation. Les miels étudiés sont moins acides que les miels récoltés de 2011 à 2012 dans l'arboretum de l'école nationale agronomique de Yamoussoukro (42,6 mEq/Kg à 43,53 $\mathrm{mEq} / \mathrm{Kg}$ ) par Iritié et al. (2014). L'acidité est un bon critère d'évaluation de la qualité du miel car elle donne des indications importantes sur son état (Bogdanov et al., 1999 ; Mezhoud, 2013). Selon ces auteurs, l'acidité du miel augmente avec la fermentation.

\section{Densité des miels}

La densité moyenne $(1,42 \pm 0,07)$ des miels analysés est similaire à celle du Codex alimentarius (1,42) pour les densités de 1,39 et 1,44 (Codex alimentarius, 2000; 2001; Abdulaziz et al., 2012). La densité du miel renseigne sur sa teneur en eau. En effet, les miels avec des densités comprises entre 1,39 et 1,44 ou supérieures ont une teneur d'eau acceptable et propice à la conservation contrairement aux miels de densités inferieures à 1,39. Le miel E4 avec une densité $(1,33)$ inférieure à 1,39 est plus riche en eau $(25 \pm 2,4)$ que les autres miels. Donc, le miel E4 pourrait vite se fermenter par rapport aux autres miels. Par contre, le miel E5 avec une densité $(1,5)$ supérieure à 1,44 a présenté une teneur en eau moins élevée. Par conséquent, le miel E4 pourrait avoir une longue durée de conservation comparativement autres miels. Ces densités sont supérieures à celles des miels des localités de Séguéla $(1,11 ; 1,1$; $1,22)$ et celle du supermarché du centre commercial de la ville de Daloa $(1,06)$ trouvées par Diomandé et al. (2018) et du miel en provenance de l'Algérie $(1,28)$ selon Djonwan $(2018)$. Des densités supérieures à la valeur maximale $(1,44)$ du Codex alimentarius ont été également enregistrées dans les miels de Danané $(1,59)$; Dimbokro $(1,55)$; Katiola $(1,63)$ et Korhogo $(1,65)$ selon Coulibaly (2014). Ces variations s'expliqueraient par la période de récolte des miels. En effet, les miels récoltés précocement des ruches sont moins dense et plus riche en eau que les miels récoltés à maturité.

\section{Teneur en eau ou humidité des miels}

La teneur en eau des miels oscille de $17 \pm 1,6 \%$ à $25 \pm 2,4 \%$ avec une moyenne de 20,2 $\pm 3,11 \%$. La plupart des miels analysés ont une teneur en eau inférieure à $21 \%$. La teneur en eau est un paramètre important à la conservation du miel. Il permet d'estimer le niveau de maturité des miels, renseigne sur la stabilité du miel contre la fermentation et la cristallisation (Mezhoud, 2013). Selon Schweitzer (2019), un miel pauvre en eau se conserve 
plus longtemps qu'un miel riche en eau quel que soit sa teneur en levure. Le miel de Mitichi (E4) avec une teneur en eau supérieure à 21\%, serait difficile à être conservé sans risque de fermentation. Les variations des teneurs en eau des miels seraient liées à la période de récolte des miels ou encore à leurs origines florales. En effet, des miels monofloraux auraient une teneur en eau élevée du fait de leurs origines botaniques : cas du miel de trèfle (Trifolium) et de bruyères (Calluna) en Europe (Codex, 2000 ; 2001).

\section{Degré Brix ou teneur en sucre totale des miels}

La teneur en sucre totale des miels se situe entre $76,50 \pm 0,10 \%$ et 81,40 $\pm 0,00 \%$. Ces valeurs sont similaires à celles obtenues dans les miels des autres régions de la Côte d’Ivoire. Dans les miels de Danané (76,58\%), Dimbokro (78,05\%), Katiola $(77,90 \%)$ et Korhogo (80,18\%) selon Coulibaly (2014). Dans les miels récoltés de 2011 à 2012 dans l'arboretum de l'école nationale agronomique de Yamoussoukro (74\% à 81\%) selon Iritié et al. (2014). Dans le miel de la coopérative de Toumodi $(75,23 \%)$ selon Assi-Kaudjhis et al. (2021). Dans les miels de Bouaflé (79\%), Djekanou (78\%) et dans des miels importés de France (80,5\%), d'Espagne (80,5\% ; 81\%), d'Amérique (80,5\% ; 82\%), d’Algérie (70\%), du Sénégal (79\%) selon Djonwan (2018). Toutes ces valeurs sont supérieures à celles du Codex alimentarius (2000; 2001). Les teneurs en sucre des miels seraient liées à leurs origines florales par la concentration en sucre des fleurs butinées par les abeilles ou à un nourrissage des abeilles au sucre pendant la miellée. Etant donné que les miels analysés proviennent de la cueillette et des ruches de la réserve où les abeilles n’ont pas été nourries au sucre, cette teneur en sucre serait liée aux nectars des fleurs butinées.

\section{Teneur en centre des miels}

La teneur en cendre des miels varie de $0,09 \pm 0,00 \%$ à $0,36 \pm 0,02 \%$. Ces valeurs sont conformes à la norme Codex alimentarius. La teneur en cendre permet d'apprécier la teneur en matières minérales ou non solubles étrangères au miel. Les miels analysés ne contiendraient pas de matières minérales ou éléments étrangers. Cependant, ces valeurs sont supérieures à celles obtenues par Iritié et al. (2014) (0,08 à 0,09\%) dans les miels de l'arboretum de l'école nationale agronomique de Yamoussoukro. Ces variations pourraient être liées à l'origine florale des miels ou à la nature des sols. Car selon Shahnawaz et al. (2013), la teneur en cendre des miels varie en fonction des sols des plantes mellifères, aux conditions atmosphériques, et à la physiologie des plantes. Les types de sols déterminent les végétations et sont propices à des cultures. En outre, ces variations se justifieraient par la différence des sols des zones de collecte. En zone forestière en Côte d'Ivoire l'on rencontre principalement les sols ferrallitiques favorables aux cultures 
d'exportations et en zone savanicole les sols ferrugineux avec une dominance de graminées.

\section{Conductivité électrique ( $\sigma)$ des miels}

La conductivité électrique des miels se situe entre 53,80 $\pm 0,17 \mu \mathrm{S} / \mathrm{cm}$ et 129,47 $\pm 0,38 \mu \mathrm{S} / \mathrm{cm}$. La conductivité électrique est un paramètre efficace de détermination de l'origine botanique des miels. Elle est désignée lors des contrôles de routine en remplacement de la teneur en cendre. Les miels analysés ont une conductivité électrique inférieure à $0,8 \mathrm{mS} / \mathrm{cm}$, ce qui confirme leur provenance du nectar. Ces valeurs sont inférieures à celles (596 $\mu \mathrm{S} / \mathrm{cm}-707 \mu \mathrm{S} / \mathrm{cm}$ ) des miels récoltés de 2011 à 2012 dans l'arboretum de l'école nationale agronomique de Yamoussoukro (Iritié et al., 2014). Ces variations s'expliqueraient par les types de plantes butinées pour élaborer le miel, par la situation phytogéographique ou aux conditions environnementales du milieu (Fechner et al., 2016).

\section{Conclusion}

Les miels analysés sont issus des localités de la Sous-Préfecture de Cechi (Département d'Agboville) en zone forestière de la Côte d'Ivoire. Ils sont généralement acides et ne sont pas exposés aux extrêmes fermentations. Ces miels pourraient être conservés longtemps sans risque de fermentation. Ce sont des miels de nectar riche en sucre qui ne contiennent pas d'éléments minéraux indésirables.

Cette étude pourrait s'étendre à l'analyse d'autres paramètres tels que la microbiologie, la recherche de métaux lourds et la mellissopalynologie. Elle devrait également s’étendre aux miels des autres régions forestières.

\section{References:}

1. Abdallah L et Saber Fatiha., 2017, Contribution à l'identification de l'origine botanique de quelques miels de la willaya de TiziOuzou par une analyse pollinique au microscope optique. Mémoire de fin d'études, en vue de l'obtention du diplôme de Master en Sciences Agronomiques. Université Mouloud Mammeri de TiziOuzou (Algérie), 135p.

2. Abdulaziz S, Alqarni, Ayman A, Owayss A, Owad A et Mahoud., 2012, Physicochemical characteristics total phenols and pigments of national and international honey in Saudi Arabia, Arabia journal of chemistry, 2012, 4, 5.

3. Achour H et Khali M., 2014, Composition physicochimique des miels algériens: Détermination des éléments traces et des éléments potentiellement toxiques. Afrique Sci.10: 27-36. 
4. Addinsoft., 2020, Tests de Bartlett et de Levene pour comparer des variances. Copyright 2020 Addinsoft.

5. Agbagwa O E, Otokunefor T V et Peterside-Frank N., 2011, Quality assessment of Nigeria honey and Manuka honey. J. Microbiol. Biotech. Res, 1 (3) : 20-31.

6. Ahouandjinou T B, Yedomonhan H, Adomou A C, Tossou M G, Akoegninou A., 2016, Caractéristiques techniques et importance socio-économique de l'apiculture au nord-ouest du bénin : cas de la commune de Cobly. Université d'Abomey-Calavi (UAC), 01 bp 4521 Cotonou, Bénin.

7. Amri A., 2006, Evaluation physico-chimique et détermination de l'origine botanique de quelques variétés de miel produites à l'Est d’Algérie. Mémoire de master, Université Badji Mokhtar-Annaba 97p.

8. Assi-Kaudjhis C, Bolou G E-K et Ouattara S., 2021, Melissopalynological and physico-chemical analysis of honey from the beekeeping cooperative of Toumodi (Côte d'Ivoire), International Journal of Scientific and Research Publications, Volume 11, Issue 2 February 2021 ISSN 2250-3153.

9. Assi-Kaudjhis C, Ebah E A et Savadogo S., 2020, Uses of Beehive Products by the Baoule Population, Central Côte d'Ivoire. International Journal of Science and Research (IJSR) ISSN: 23197064 SJIF (2019).

10. Aubréville A., 1958, Flore forestière soudano-guinéenne. Soc d’Ed. Géogr. Mar. et Col. Pari (France), 523 p.

11. Bogdanov S, Lullman C, Martin P, Von Der Hoe W, Rusmann H, Vorwohl G, Persano-Oddo L, Sabatini A G, Marcazzwan G L, Piro R, Flamini C, Marlo M, Hertier J, Borneck R, Marioleas P, Tsigouri A, Kerkvliet J, Ortiz A, Ivanov T, Arcy D B, Mossel B et Vit P., 1999, Honey quality and international regulatory standards, review by international honey commission, Bee world 80 (2) 1999, 61-69.

12. Bradbear N., 2005, Apiculture et moyens d'existence durables. Organisation des nations unies pour l'alimentation et l'agriculture. ISSN 1813-6001, Rome, $64 \mathrm{p}$.

13. Codex A., 2000, Programme mixte FAO/OMS sur les normes alimentaires. Comité du Codex sur les sucres, septième session, Londres, Royaume-Uni, 9- 11 Février 2000.

14. Codex A., 2001, Programme Mixte FAO/OMS Sur Les Normes Alimentaires. Commission du Codex Alimentarius. ALINORM, 25 (1), 1-31. 
15. Coulibaly., 2014, Potentialités de production mellifère de la flore de transition foret savane, en zone guinéenne et caractéristique physicochimique de quelques miels de la Côte d'Ivoire. Thèse de doctorat, Université Felix Houphouët Boigny (Côte d'Ivoire) 194p.

16. Diomandé M, Coulibaly S, Koko A C et Bahoua L G., 2018, Identification des plantes mellifères et propriétés physicochimiques de miels de la région du Worodougou, Cote d'Ivoire. International Jounal of Current Research vol 10, issue, 04, pp 67583-67590 April 2018.

17. Djonwan F D., 2018, Etude comparative de la qualité des miels locaux et importés en Côte d'Ivoire. Thèse de diplôme d'Etat de docteur en pharmacie de Université Felix Houphouët Boigny 82 p.

18. Fechner D C, Mores A L, Riuz Diaz J D, Pellerano R G et Vazquez F A., 2016, Multivariate classification of honeys from Carrientes (Argentina) according to geographical origin based on physicochemical properties. Food Biosci. 15 : 49-54.

19. INS., 2014, Recensement général de la population et de l'habitat de la Côte d'Ivoire. Institut national de la statistique. Document non publié.

20. Iritié M B, Eboua N W, Yapo Y M, Fantodji A et Bodji C N., 2014, Comparative analysis physicochemical characteristics of honeys produced in the multi-flora arboretum of the national school of agronomy of Yamoussoukro. International journal of agricultural policy and research vol.2 (11) pp. 379-382, November 2014.

21. Julika N W, Ajit A, Sulaiman A Z et Naila A., 2019, Physicochemical and Microbiological Analysis of Stingless Bees Honey Collected from Local Market in Malaysia.indones. J. Chem. 19 (2): 522-530.

22. Kombo P., 1989, Apiculture et miel dans la province de l'Adamaoua (Cameroun). Thèse de doctorat 196p.

23. Kouame K F, Akpesse A A, Diby Y S et Kassi N J., 2021, Morphometric characteristics of honeybees in the cechi nature reserve in the department of agboville (south-east, Ivory Coast). International Journal of Food Science and Nutrition, Volume 6, Issue 3, 2021, Page No. 120-127.

24. Kouamé K F, Assi K C, Kiyinlma C, Kassi J., 2020, Inventaire et identification des plantes melliferes de la zone guineenne : cas de la foret Yapi Daniel et extension (Sud de la Cote d'Ivoire). European Scientific Journal, ESJ, 16 (33), 315.

25. Koudegnan C, Etse K, Coulibaly S, Quashie M-L, Radji P, Kokou K., 2021, Caractérisations physico-chimiques des miels de la zone 
Guinéenne du Togo. Revue Marocaine des Sciences Agronomiques et Vétérinaires p-ISSN: 2028-991X

26. Louveau J., 1985, Les miels d'Afrique du Nord et leur spectre pollinique. Apidologie pp : 160-1970.

27. Maglon G et Vanwijek R., 2003, Guide des plaies. Ed.J.L. Eurotext, Paris, France, 102 p.

28. Makhloufi C, Kerkvliet J D, Ricciardelli D’albore G, Choukri A et Samar R., 2013, Characterization of Algerian honeys by palynological and physico-chemical methods. Apidologie, 41 : 509-521.

29. Makhtar D., 2002, La filière apicole au Sénégal. Thèse présentée et soutenue publiquement le 15 mai 2002 devant la faculté de Médecine, de Pharmacie et d'Odonto-stomatologie de Dakar pour obtenir le grade de Docteur vétérinaire, Sénégal 140p.

30. Mehrnaz K-S et Bonmatin J-M., 2003, Usage du miel dans les médicaments simples et composés chez Rhazès, $91^{\mathrm{e}}$ année, n³37,2003. pp. 29-36.

31. Mezhoud I., 2013, Analyse physicochimique et étude de l'adultération de miel de la région de Bejaia. Mémoire de master, Université A. MIRA- Bejaia- Faculté des Sciences Exacte, Département de Chimie, Algérie 48p.

32. Moussaoui N., 2011, Analyse sensorielle de quelques miels de Sud Algérien. Mémoire de fin d'étude, Université Kasdi MerbahOuargba, Algérie.

33. N’Guessan K, Kadja B, Zirihi G N, Traoré D et Aké-Assi L., 2009, Screening phytochimiques de quelques plantes médicinales ivoiriennes utilisées en pays Krobou (Agboville, Côte-d'Ivoire). Sci. Nat., 6(1) : 1-15.

34. Randrianarivelo R H M., 2010, Application de l'analyse pollinique aux miels de différentes régions de Madagascar. Mémoire de DEA, Université d'Antananarivo, Madagascar 71p.

35. Rebiai A, Lanez T et Chouikh L., 2015, Physicochemical and biochemical properties of honeybee products in South Algeria.St. Cerc. St. CICBIA. 16 (2), 133-142.

36. Schweitzer P., 2019, Les critères de qualité du miel : Article $<<$ sur les sentiers des miels de France>> avec l'autorisation de "l'abeille de France"

37. Selvaraju K, Vikram P, Soon JM, Krishnan K T et Mohammed A., 2019, Mellissopalynological, physicochemical and antioxidant properties of honey from West Coast of Malaysia. J Food Sci Technol. 56 (5): 2508-2521. 
38. Shahnawaz M, Saghir A S, Mirza H, Abdul R et Sadat S K., 2013, A study on the determination of physicochemical properties of honey from différent valleys of Gilgit-Baltistan. International Journal of Agricultural Science Research, 2(2) : 049-053. 